\title{
Upper lip tie wrapping into the hard palate and anterior premaxilla causing alveolar hypoplasia
}

\author{
Woong Heo, \\ Hee Chang Ahn \\ Department of Plastic and Reconstructive \\ Surgery, Hanyang University Hospital, Seoul, \\ Korea
}

\begin{abstract}
Bony anomaly caused by lip tie is not many reported yet. There was a case of upper lip tie wrapping into the anterior premaxilla. We represent a case of severe upper lip tie of limited lip motion, upper lips curling inside, and alveolar hypoplasia. Male patient was born on June 3,2016 . He had a deep philtral sulcus, low vermilion border and deep cupid's bow of upper lip due to tension of short, stout and very tight frenulum. His upper lip motion was severely restricted in particular lip eversion. There was anterior alveolar hypoplasia with deep sulcus in anterior maxilla. Resection of frenulum cord with Z-plasty was performed at anterior premaxilla and upper lip sulcus. Frenulum was tightly attached to gingiva through gum and into hard palate. Width of frenulum cord was about $1 \mathrm{~cm}$, and length was about $3 \mathrm{~cm}$. He gained upper lip contour including cupid's bow and normal vermilion border after the surgery. This case is severe upper lip tie showing the premaxillary hypoplasia, abnormal lip motion and contour for child. Although there is mild limitation of feeding with upper lip tie child, early detection and treatment are needed to correct bony growth.
\end{abstract}

Keywords: Labial frenum / Maxilla / Diastema / Breast feeding

\section{INTRODUCTION}

Upper lip tie is uncommon case which makes it difficult for babies to feed breast milk. It is revealed by researches that upper lip tie and tongue tie can make functional problem, but almost no one had bony anomaly caused by lip tie [1-5]. And in most cases, it is not treated if there is no limitation of baby's growing result for difficulty of breastfeeding [1,2]. There was a case of upper lip tie wrapping into the anterior premaxilla in our clinic. It was tight cord of lip strongly attached from vermilion to premaxilla. We checked up facial three-dimensional computed tomography (3D CT) and found that there was an alveolar hypoplasia caused by severe upper-lip tie. We represent a case of particular upper lip tie showing limited lip motion, upper lips curling inside, and alveolar hypoplasia. It was needed to be released for alveolar growing and lip contour.

Correspondence: Hee Chang Ahn

Department of Plastic and Reconstructive Surgery, Hanyang University Hospital, 222-1

Wangsimni-ro, Seongdong-gu, Seoul 04763, Korea

E-mail: ahnhc@hanyang.ac.kr

Received January 24, 2018 / Revised March 13, 2018 / Accepted March 14, 2018

\section{CASE REPORT}

Male patient was born on June 3, 2016 without choromosomal abnormality. He had a deep philtral sulcus, low vermilion border and deep cupid's bow of upper lip due to tension of short, stout and very tight frenulum. His sucking power was almost normal, but upper lip motion was severely restricted in particular lip eversion from anterior maxilla. We checked up facial 3D CT so that there was anterior alveolar hypoplasia with deep sulcus inside. Because his upper lip tie was toughly attached through hard palate and anterior maxilla, we thought it possibly made alveolar hypoplasia (Fig. 1). We tried to detect any other accompanied congenital malformation, but it was not classified any other congenital disease. We decided to release tie to make him have adequate alveolar and premaxillar growth and lip motion including lip contour. Eight-month-old patient admitted for frenulectomy with Z-plasty of frenulum through upper lip and premaxilla. Resection of frenulum cord with Z-plasty was successfully performed at anterior premaxilla and upper lip sulcus. Frenulum was 


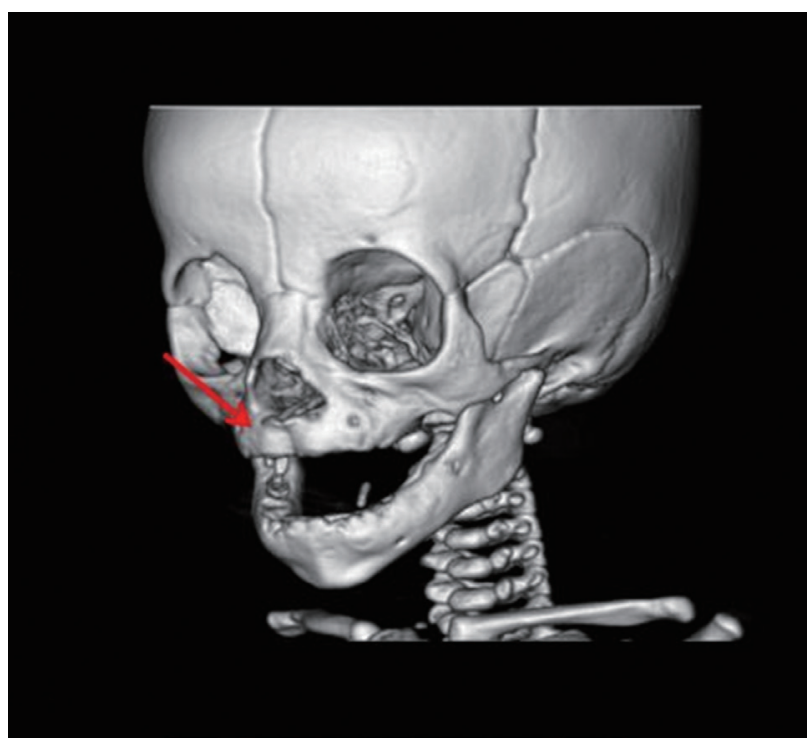

Fig. 1. Preoperative facial three-dimensional computed tomography. It shows the bony sulcus of premaxilla and alveolus due to tight, short cord of upper lip frenulum (arrow).

tightly attached to gingiva through gum and into hard palate. Width of frenulum cord was about $1 \mathrm{~cm}$ and length was about 3 $\mathrm{cm}$. Cord was too wide and tough, and it caused the deficiency of alveolus bone between upper incisors, probably causing oral cavity anomaly from childhood (Fig. 2). He gained upper lip contour including cupid's bow and normal vermilion border after the surgery (Fig. 3). We rechecked facial 3D CT and found that alveolar hypoplasia was almost corrected at postoperative 10 months (Figs. $4,5)$.

\section{DISCUSSION}

Upper lip tie is a benign condition that tends to improve with normal facial growth [6]. It can be contributing factor to breastfeeding difficulty and abnormal lip motion. It may cause ineffective latching but significant functional problem like speech production is not reported yet [1]. Generally, relief surgery for upper lip tie may be indicated when the baby has not enough sucking ability for breast feeding or abnormal lip motion [3]. But there are several cases that upper lip tie alone can cause maxillary diastema, or gap between upper two central teeth [1].

It is reported that frenulotomy of upper lip tie alone results in

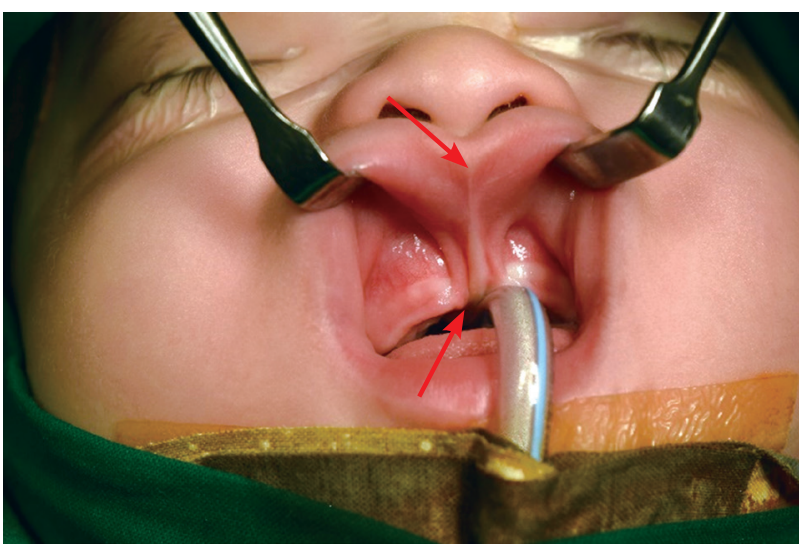

Fig. 2. Intraoperative photograph. There was stout frenulum cord wrapped gum into the anterior premaxilla, and invaded into the alveolus. Eversion of lip was not possible due to the cord tightness (arrows).

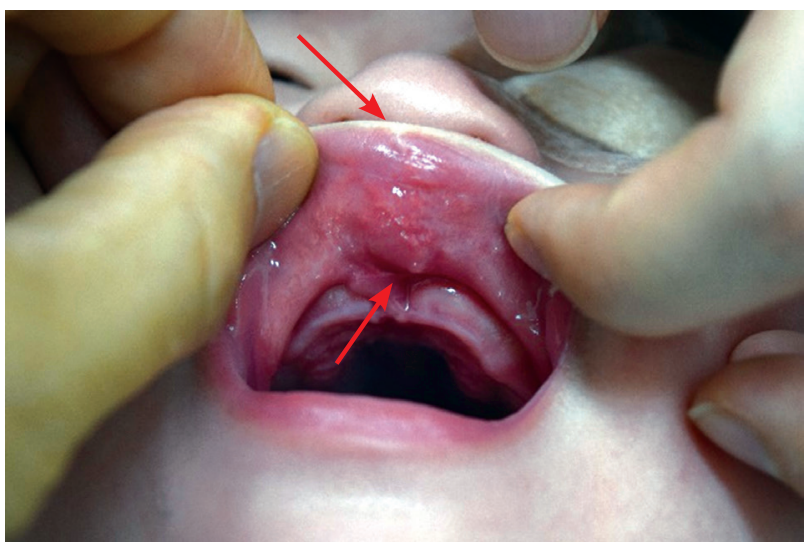

Fig. 3. Postoperative 2 months and 17 days photograph. Cord was resected from premaxilla and gum. Lip was completely released from the alveolus so that upper lip can be everted well after surgery (arrows).

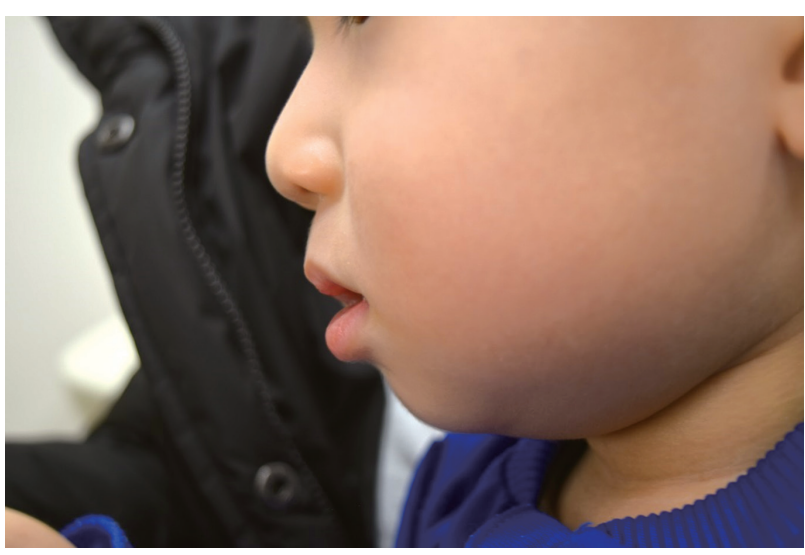

Fig. 4. Postoperative 10-month photograph. Natural upper lip contour after lip release at postoperative 10 months (lateral side). 


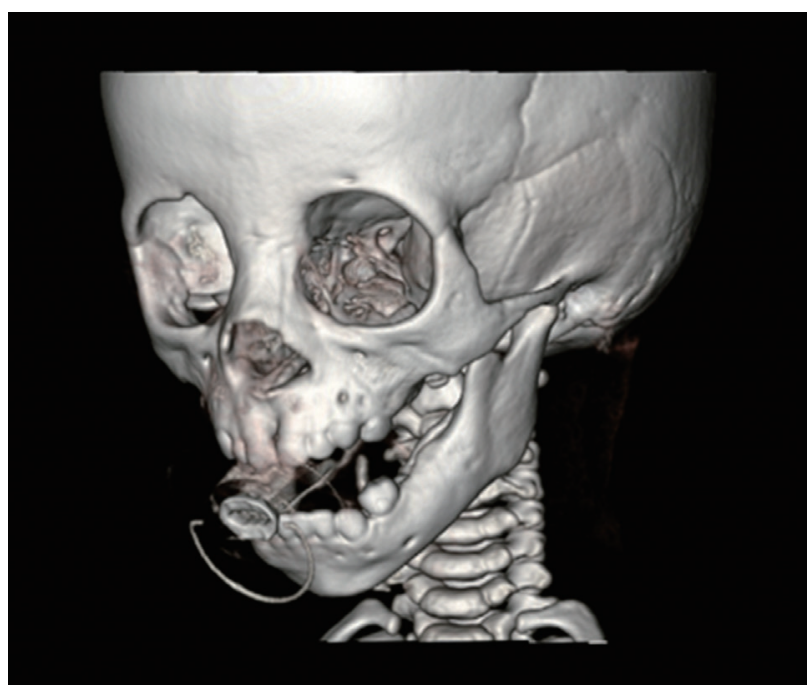

Fig. 5. Postoperative 10-month facial three-dimensional computed tomography. It shows the bony growth of premaxilla and alveolus compared to preoperative computed tomography.

low recurrence rate and high improvement rate of breast feeding $[1,3,7]$. For mild upper lip tie, simply dividing of frenulum by iris scissor can be the treatment of choice [7]. But this particular case is severe upper lip tie resulting alveolar hypoplasia, abnormal lip motion and contour for child. And it is very rare and needed to release the cord. In this case, upper lip was curling inside because of cord tightness. Thus, we should release inner upper lip by Zplasty after cord resection.

This case demonstrates that tension of upper lip tie itself can make bony hypoplasia and abnormal lip contour for child. Although there is no limitation of feeding with upper lip tie child, early detection and treatment are needed to correct bony growth. Severe case of upper lip tie like in this case, cord resection with releasing by Z-plasty can be successful surgical technique. We should closely observe recurrence and maxillary growth in his growing period.

\section{CONFLICT OF INTEREST}

No potential conflict of interest relevant to this article was reported.

\section{PATIENT CONSENT}

The patients provided written informed consent for the publication and the use of their images.

\section{REFERENCES}

1. Pransky SM, Lago D, Hong P. Breastfeeding difficulties and oral cavity anomalies: the influence of posterior ankyloglossia and upper-lip ties. Int J Pediatr Otorhinolaryngol 2015;79:1714-7.

2. Ghaheri BA, Cole M, Fausel SC, Chuop M, Mace JC. Breastfeeding improvement following tongue-tie and lip-tie release: a prospective cohort study. Laryngoscope 2017;127:1217-23.

3. Kotlow LA. Diagnosing and understanding the maxillary lip-tie (superior labial, the maxillary labial frenum) as it relates to breastfeeding. J Hum Lact 2013;29:458-64.

4. Popovich F, Thompson GW, Main PA. The maxillary interincisal diastema and its relationship to the superior labial frenum and intermaxillary suture. Angle Orthod 1977;47:265-71.

5. Gkantidis N, Kolokitha OE, Topouzelis N. Management of maxillary midline diastema with emphasis on etiology. J Clin Pediatr Dent 2008;32:265-72.

6. Dewel BF. The labial frenum, midline diastema, and palatine papilla: a clinical analysis. Dent Clin North Am 1966:175-84.

7. Benoiton L, Morgan M, Baguley K. Management of posterior ankyloglossia and upper lip ties in a tertiary otolaryngology outpatient clinic. Int J Pediatr Otorhinolaryngol 2016;88:13-6. 